\title{
PROMOÇÃO DO DESENVOLVIMENTO INFANTIL NA ESCOLA ATRAVÉS DE ATIVIDADES MOTORAS RECREATIVAS
}

\author{
Raysa Maldonado Lima \\ Universidade Federal de Santa Maria \\ raysa_ml_113@hotmail.com
}

\author{
Marina Segala \\ Universidade Federal de Santa Maria \\ marina_sgl@hotmail.com \\ Claudia Morais Trevisan \\ Universidade Federal de Santa Maria \\ claudiamoraistrevisan@gmail.com
}

\begin{abstract}
Resumo
A ação de extensão teve como objetivos: investigar o perfil do desenvolvimento motor, propor atividades recreativas que estimulassem a aquisição e aperfeiçoamento das habilidades motoras fundamentais e analisar o impacto da mudança no plano curricular pedagógico da escola. As atividades foram realizadas uma vez por semana no período de 2010 a 2013 na Escola Municipal de Educação Infantil Ady Schneider Beck, Santa Maria, RS. Participaram da ação crianças de 03 a 06 anos, matriculadas regularmente na escola e com TCLE assinado pelo responsável. Após a proposta de estimulação, houve um ganho em $74 \%$ de aperfeiçoamento nas atividades motoras quando comparadas à avaliação inicial. A ação de extensão auxiliou no desenvolvimento neuromotor de crianças com atrasos, refletindo na implementação das atividades do projeto no currículo pedagógica da escola, elucidando a importância da extensão universitária.

Palavras-chave: Extensão Universitária. Desenvolvimento Neuromotor Infantil. Estimulação Motora.
\end{abstract}

\section{PROMOTION OF CHILD DEVELOPMENT AT SCHOOL THROUGH MOTOR ACTIVITIES RECREATIONAL}

Abstract

The extension action aimed to: investigate the motor development profile, propose recreational activities that stimulate the acquisition and improvement of fundamental motor skills and analyze the impact of the change in the political pedagogical curriculum of the school. The activities were held once a week in the period from 2010 to 2013 at the Municipal School of Early Childhood Education Ady Schneider Beck, Santa Maria, RS. In the project participated children 03-06 years old, regularly enrolled in school and with informed consent signed by a responsible. After the proposal of stimulation, there was a gain in $74 \%$ improvement in motor skills when compared to baseline. The extension action helped in the neuromotor development of children with delays, reflecting in the implementation of the project activities in the school's educational curriculum, explaining the importance of university extension.

Keywords: University Extension. Infant Neuromotor Development. Motor Stimulation.

\section{PROMOCIÓN DEL DESARROLLO INFANTIL EN LA ESCUELA A TRAVÉS DE ACTIVIDADES MOTORAS RECREATIVAS}

\section{Resumen}

La acción de extensión tuvo como objetivos: investigar el perfil de desarrollo motor, ofrecer actividades recreativas que estimulen la adquisición y mejora de las habilidades motoras fundamentales y analizar el impacto del cambio en el plan de estudios pedagógicos de la escuela. Las actividades se han realizado una vez por semana en el período 2010 a 2013 en la Escuela Municipal de Educación Infantil Ady Schneider Beck, Santa Maria, RS. Participaronde la acción niños de 03-06 años de edad, matriculados regularmente en la escuela y con el consentimiento informado firmado por la persona responsable. Después de la estimulación propuesta, hubo un aumento en $74 \%$ de perfeccionamiento en las habilidades motoras en comparación con el valor basal. La acción de extensión ayudó a ganar el desarrollo neuromotor de los niños con retrasos, reflexionando en la implementación de las actividades del proyecto en el currículo educativo de la escuela, aclarando la importancia de la extensión universitaria.

Palavras clave: Extensión Universitaria. Desarrollo Neuromotor Infantil. Estimulación Motriz. 


\section{INTRODUÇÃO}

A extensão universitária é definida como um processo educativo, cultural e científico que articula o ensino e a pesquisa de forma indissociável e viabiliza a relação transformadora entre universidade e sociedade (NOGUEIRA, 2000).Esse processo envolve a comunidade, inclui o trabalho interdisciplinar e favorece a visão integrada do social (PONTE et al., 2009).

Ações de extensão que visem à promoção e à prevenção da saúde podem ser realizadas em hospitais, Unidades Básicas de Saúde, Centros de Ensino e na própria comunidade, podendo atingir assim, uma maior parcela da população (CASATE e CORRÊA, 2005).

Essas ações quando realizadas em ambientes escolares, principalmente entre crianças da primeira infância, garantem os direitos à saúde, educação e cidadania garantidos pelo Estatuto da Criança e do Adolescente pela Lei de Diretrizes e Bases da Educação Nacional, permitindo a criação de condições favoráveis ao desenvolvimento integral dessa parcela da população (RIZZETTTI et al., 2009).

O potencial de uma criança é definido pelas suas experiências durante os primeiros anos de vida. Se esse período não for aproveitado, as possibilidades de desenvolvimento ficarão prejudicadas (FERREIRA et al., 2002). A infância é o período no qual os momentos críticos do processo de desenvolvimento são facilmente detectáveis, sendo esse um aspecto relevante para o pesquisador da área do desenvolvimento motor, no momento de escolher as etapas iniciais da vida para testar suas hipóteses (SANTOS et al., 2004).

O desenvolvimento motor pode ser considerado um processo sequencial, contínuo, relacionado à idade cronológica e aos fatores ambientais. O ser humano sofre processos de alterações nos níveis de funcionamento e adquire uma enorme quantidade de habilidades motoras, as quais progridem de movimentos simples e desorganizados, para altamente organizados e complexos (MAFORTE et al., 2007; WILLRICH et al., 2009; TORQUATO et al., 2011).

A idade pré-escolar, compreendida entre os dois e seis anos de idade, é a fase de aquisição e aperfeiçoamento das habilidades motoras fundamentais (CAETANO et al., 2005). Assim, um atraso no desenvolvimento motor durante essa fase acarretará prejuízos futuros na vida social das crianças (TORQUATO et al., 2011).

Segundo, Gallahue \& Ozmun (2005) os padrões fundamentais de movimento são observados a partir dos dois anos, até aproximadamente sete anos. Esses movimentos são 
categorizados como estabilizadores locomotores e manipulativos e classificados em estágio inicial, elementar e maduro.

O estágio inicial, típico aos 02 anos de idade, representa as habilidades fundamentais aonde o movimento é caracterizado por elementos que faltam ou que são sequenciados e restritos, pelo uso exagerado do corpo, por fluxo rítmico e por coordenação deficiente. O estágio elementar, típico aos 03 ou 04 anos, envolve maior controle e melhor coordenação rítmica dos movimentos fundamentais. Aprimora-se a sincronização dos elementos temporais e espaciais do movimento, porém, os padrões de movimento ainda são restritos ou exagerados, embora mais bem coordenados. O estágio maduro na fase de movimentos fundamentais é caracterizado por desempenhos mecanicamente eficientes, coordenados e controlados que deveriam acontecer aos 05 ou 06 anos de idade.

Segundo Lopes et al. (2011) o movimento serve como janela para o processo de desenvolvimento motor, porém o ambiente e as condições de vida atuais tornam difícil para muitas crianças adquirirem experiências motoras, variadas. Sendo assim, a falta de movimento pode não só levar a um restringir do corpo e do desenvolvimento motor, como pode influenciar aspectos da personalidade como a percepção, a cognição, o discurso, as emoções, e o comportamento social.

Dessa forma os objetivos da ação de extensão foram investigar o perfil do desenvolvimento motor, propor atividades recreativas que estimulem a aquisição e aperfeiçoamento das habilidades motoras fundamentais e analisar o impacto da mudança no plano curricular pedagógico da escola.

\section{MATERIAIS E MÉTODOS}

A ação de extensão foi desenvolvida na Escola Municipal de Educação Infantil (EMEI) Ady Schneider Beck, localizada na Vila Berleze, área distrital do SUS, relacionada à Universidade Federal de Santa Maria, na cidade de Santa Maria, Rio Grande do Sul. Essa beneficia aproximadamente 80 alunos com idades entre 02 e 06 anos, divididos em cinco turmas nos turnos da manhã e tarde.

O primeiro contato ocorreu no ano de 2010 com a finalidade de explanar aos professores da escola os objetivos da ação de extensão. Essa era intitulada "Prevenção de Distúrbios Neuropsicomotores em Crianças nos Centros Municipais de Educação Infantil no Município de 
Santa Maria, RS" (UFSM/CCS/SIE $n^{\circ}$ de registro 026253) teve como objetivos: diagnosticar o desenvolvimento neuropsicomotor em crianças regularmente matriculadas na instituição de ensino; implementar uma proposta de estimulação neuromotora em crianças com sinais de alterações no plano instrumental do desenvolvimento; sensibilizar a equipe da unidade, quanto a sua importância na promoção do desenvolvimento integral da criança; proporcionar orientação às famílias das crianças que frequentam a escola, sobre a importância de sua participação no desenvolvimento da criança; solicitar avaliação dos diferentes profissionais da rede básica de saúde para as crianças em situação de risco; e encaminhar ao Serviço de Fisioterapia do Hospital Universitário de Santa Maria as crianças que necessitassem de intervenção terapêutica.

Os critérios de inclusão foram ter entre 03 e 06 anos e estar matriculado regularmente na escola, na qual os responsáveis assinaram um termo que permitia a realização de atividades de ensino e extensão, aprovados previamente nas respectivas unidades de ensino. Os critérios de exclusão foram os alunos somarem mais de $25 \%$ de faltas às atividades de estimulação.

Depois de esclarecidos os objetivos e assinados os Termos de Consentimento Livre e Esclarecido (TCLE) iniciaram-se no ano de 2011 as visitas para avaliação motora dos alunos. Essas ocorreram uma vez por semana em cada turno (manhã e tarde), com o objetivo de identificar o estágio em que se classificavam, delinear um padrão motor geral e, assim, montar um plano de atividades para estimular o desenvolvimento motor, principalmente dos padrões que estavam abaixo do esperado.

Posteriormente à elaboração do plano de atividades, foram realizadas as visitas de estimulação, essas ocorreram uma hora por semana para cada turma, durante 08 meses nos anos de 2011, 2012 e 2013. Sendo reavaliados ao final de cada ano, visando adequar e melhorar o plano de atividades. No final do ano de 2013 foi realizada a avaliação final. Além disso, ao final de cada ano, os profissionais da escola eram convidados a participar de reuniões onde os resultados obtidos eram apresentados e dúvidas eram discutidas e esclarecidas.

O instrumento de coleta de dados foi a avaliação motora (ANEXOI) baseada na matriz de análise dos movimentos fundamentais de Gallahue; Ozmun (2005), na qual foram analisados os padrões dos movimentos estabilizadores, locomotores e manipulativos.

As brincadeiras foram selecionadas a partir de Godall (2004) e Kishimoto et al. (2011) sendo utilizadas nas avaliações e na estimulação motora, pois contemplam os movimentos avaliados, sendo essas: queimada/caçador: corrida, arremesso por cima, desvio, deslizamento; pega-pega: corrida, movimentos axiais, desvio; ovo-podre: corrida, movimentos axiais, desvio; chutar pênalti: rolamento de bola, movimentos axiais, chute, aparar a bola; basquete (acertar a 
cesta): movimentos axiais, quicar, arremesso por cima; vôlei: voleio, movimentos axiais; circuito (pular do banco, correr, pular sobre uma pequena caixa, saltar e pegar uma bola pendurada): salto em determinada altura, corrida, salto horizontal e salto vertical.

Além dessas, outras atividades, escolhidas pelos alunos e aprovadas pelo avaliador, eram utilizadas de acordo com as preferências de cada turma, com a intenção de tornar menos cansativo e mais prazeroso os momentos de avaliação e estimulação.

Os três avaliadores foram inicialmente treinados pela coordenadora da ação de extensão e realizaram todas as etapas desde a avaliação inicial, estimulação, reavaliações e avaliação final.

\section{RESULTADOS E ANÁLISES}

A análise dos resultados será apresentada de forma qualitativa e quantitativa. Entre os resultados obtidos, vale ressaltar a total adesão dos professores aos objetivos propostos. Isso ocorreu através de alterações no plano curricular político pedagógico da escola, que além das atividades de ensino tradicionais, acrescentou atividades motoras recreativas (Quadro 1 e Quadro 2).

Quadro 1 - Atividades relacionadas ao desenvolvimento motor para crianças de 0 a 03 anos

CRIANÇAS DE 00 A 03 ANOS

\begin{tabular}{|l|l|l|}
\hline \multicolumn{1}{|c|}{ OBJETIVOS } & \multicolumn{1}{|c|}{$\begin{array}{c}\text { CONTEÚDOS (grandes } \\
\text { conceitos) }\end{array}$} & \multicolumn{1}{c|}{ RELAÇÕES } \\
\hline $\begin{array}{l}\text { Familiarizar-se com a imagem } \\
\text { do próprio corpo. }\end{array}$ & $\begin{array}{l}\text { Expressividade } \\
\text { Equilíbrio e coordenação } \\
\begin{array}{l}\text { Explorar a possibilidade de } \\
\text { expres e ritmos corporais para } \\
\text { brincadeiras e nas demais } \\
\text { situações de interação. }\end{array}\end{array}$ & $\begin{array}{l}\text { Reconhecimento progressivo } \\
\text { de segmentos e elementos do } \\
\text { próprio corpo. }\end{array}$ \\
$\begin{array}{l}\text { Deslocar-se com destreza } \\
\text { progressivamente no espaço } \\
\text { ao andar, correr, pular, } \\
\text { desenvolvendo atitude de } \\
\text { confiança nas próprias } \\
\text { capacidades motoras. }\end{array}$ & $\begin{array}{l}\text { Brincadeiras; Jogos; Dança; } \\
\text { Teatro. Interação com os } \\
\text { outros. }\end{array}$ \\
$\begin{array}{l}\text { Explorar e utilizar os } \\
\text { movimentos de preensão, }\end{array}$ & $\begin{array}{l}\text { Sensações e ritmos corporais } \\
\text { gestos, posturas, linguagem } \\
\text { oral). }\end{array}$ \\
\hline
\end{tabular}




\begin{tabular}{|c|c|}
\hline $\begin{array}{l}\text { encaixe, lançamento para o } \\
\text { uso de objetos diversos. }\end{array}$ & $\begin{array}{l}\text { espaço (arrastar-se, engatinhar, } \\
\text { rolar, correr, saltar). } \\
\text { Aperfeiçoamentos dos gestos } \\
\text { relacionando com a preensão, } \\
\text { o encaixe, o traçado no } \\
\text { desenho, o lançamento. }\end{array}$ \\
\hline
\end{tabular}

Fonte: Proposta política pedagógica da EMEI Ady Schneider Beck, 2012; página 28.

Quadro 2 - Atividades relacionadas ao desenvolvimento motor para crianças de 04 a 05 anos

CRIANÇAS DE 04 E 05 ANOS

\begin{tabular}{|c|c|c|}
\hline OBJETIVOS & $\begin{array}{c}\text { CONTEÚDOS (grandes } \\
\text { conceitos) }\end{array}$ & RELAÇÕES \\
\hline $\begin{array}{l}\text { Ampliar as possibilidades expressivas } \\
\text { do próprio movimento, utilizando } \\
\text { gestos diversos e o ritmo corporal } \\
\text { nas suas brincadeiras, danças, jogos e } \\
\text { demais situações de interação. } \\
\text { Apropriar-se progressivamente da } \\
\text { imagem global do seu corpo, } \\
\text { conhecendo e identificando seus } \\
\text { segmentos e elementos e } \\
\text { desenvolvendo cada vez mais uma } \\
\text { atitude de interesse e cuidado com o } \\
\text { próprio corpo. } \\
\text { Explorar diferentes qualidades e } \\
\text { dinâmicas dos movimentos como } \\
\text { força, velocidade, resistência e } \\
\text { flexibilidade, conhecendo } \\
\text { gradativamente os limites e as } \\
\text { potencialidades do seu corpo. } \\
\text { Controlar gradualmente o próprio } \\
\text { movimento, aperfeiçoando seus } \\
\text { recursos de deslocamento e } \\
\text { ajustando suas habilidades motoras } \\
\text { para utilização em jogos, } \\
\text { brincadeiras, danças e demais } \\
\text { situações. } \\
\text { Utilizar os movimentos de preensão, } \\
\text { encaixe, lançamento, para ampliar } \\
\text { suas possibilidades de manuseio de } \\
\text { objetos. }\end{array}$ & $\begin{array}{l}\text { Expressividade } \\
\text { Equilíbrio e coordenação }\end{array}$ & $\begin{array}{l}\text { Utilização expressiva } \\
\text { intencional do movimento. } \\
\text { Brincadeiras, jogos, danças, } \\
\text { teatro, (correr, pular, subir, } \\
\text { descer, escorregar, pendurar- } \\
\text { se, movimentar-se e dançar). } \\
\text { Percepção das sensações, } \\
\text { limites, potencialidades, sinais } \\
\text { vitais e integridade do próprio } \\
\text { corpo. } \\
\text { Deslocamento e habilidades de } \\
\text { força velocidade, resistência e } \\
\text { flexibilidade. } \\
\text { Manipulação de materiais, } \\
\text { objetos, brinquedos diversos } \\
\text { para o aperfeiçoamento de } \\
\text { suas habilidades manuais. }\end{array}$ \\
\hline
\end{tabular}


Promoção do desenvolvimento infantil na escola através de atividades motoras recreativas

Fonte: Proposta política pedagógica da EMEI Ady Schneider Beck, 2012; página 28.

Ainda os educadores da escola desenvolveram um projeto intitulado "Arte em Movimento" que baseado nas atividades desenvolvidas na ação de extensão, criou e selecionou brincadeiras para estimular a aquisição de habilidades motoras. O projeto é realizado uma vez por semana, e tem como objetivos estimular o desenvolvimento motor, psicossocial e cognitivo; proporcionar maior liberdade para as brincadeiras e promover maior interação e socialização entre as diferentes turmas.

Após a análise dos dados de acordo com a matriz de Gallahue; Ozmun (2005) e considerando, de acordo com os autores, que as crianças com 02 anos apresentam-se no estágio inicial, com 03 e 04 anos no estágio elementar e 05 e 06 anos devem alcançar o estágio maduro. Pode observar-se que na avaliação inicial (Figura 1) 64\% das atividades analisadas se apresentam abaixo do esperado para a faixa etária das crianças, sendo os movimentos manipulativos como o voleio, quicar, rolamento de bola e o movimento estabilizador deslizamento, as atividades com maiores índices deficitários. Nota-se ainda que apenas 32\% das atividades estão dentro da normalidade e $4 \%$ acima do padrão para a idade. Entre as atividades a que apresentam-se com melhores índices esta o movimento locomotor caminhada.

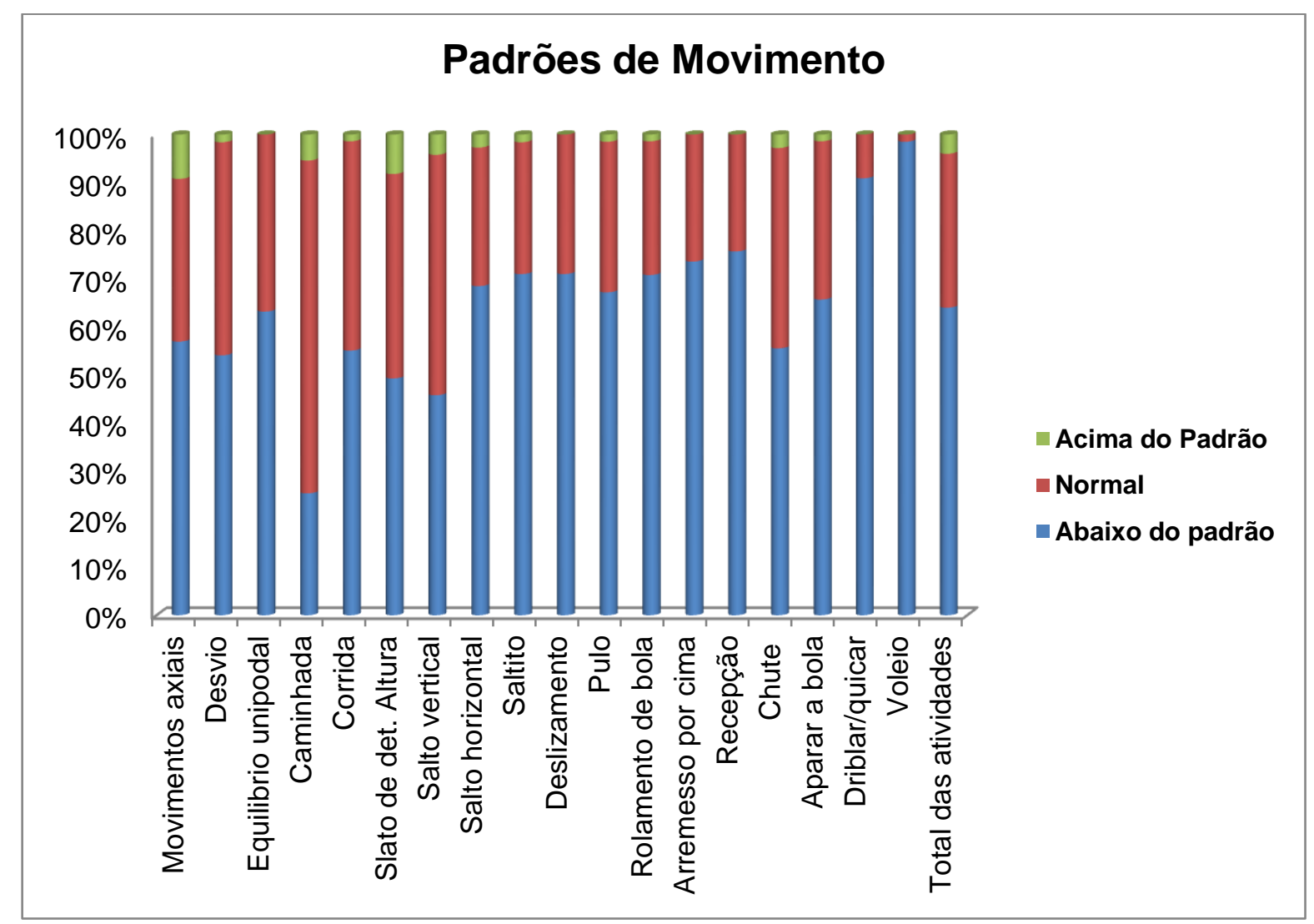

Figura 1 - Percentual da avaliação inicial do total de alunos avaliados. 
Promoção do desenvolvimento infantil na escola através de atividades motoras recreativas

$\mathrm{Na}$ avaliação final (Figura 2) observa-se que o percentual de atividades abaixo do padrão decresce $37 \%$ em relação à avaliação inicial, ficando em $27 \%$, enquanto as atividades dentro da normalidade atingiram 59\%, evidenciando um aumento de $27 \%$. Dentre as atividades analisadas o movimento manipulativo voleio, foi o que se manteve com maior percentual abaixo do padrão em ambas as avaliações, mesmo com diminuição de $50 \%$ na final. O padrão de movimento que apresentou a maior quantidade de atividades abaixo do esperado para a idade foi o manipulativo seguido do locomotor, porém na avaliação final todas as atividades se apresentaram como normal ou acima do padrão para a idade.

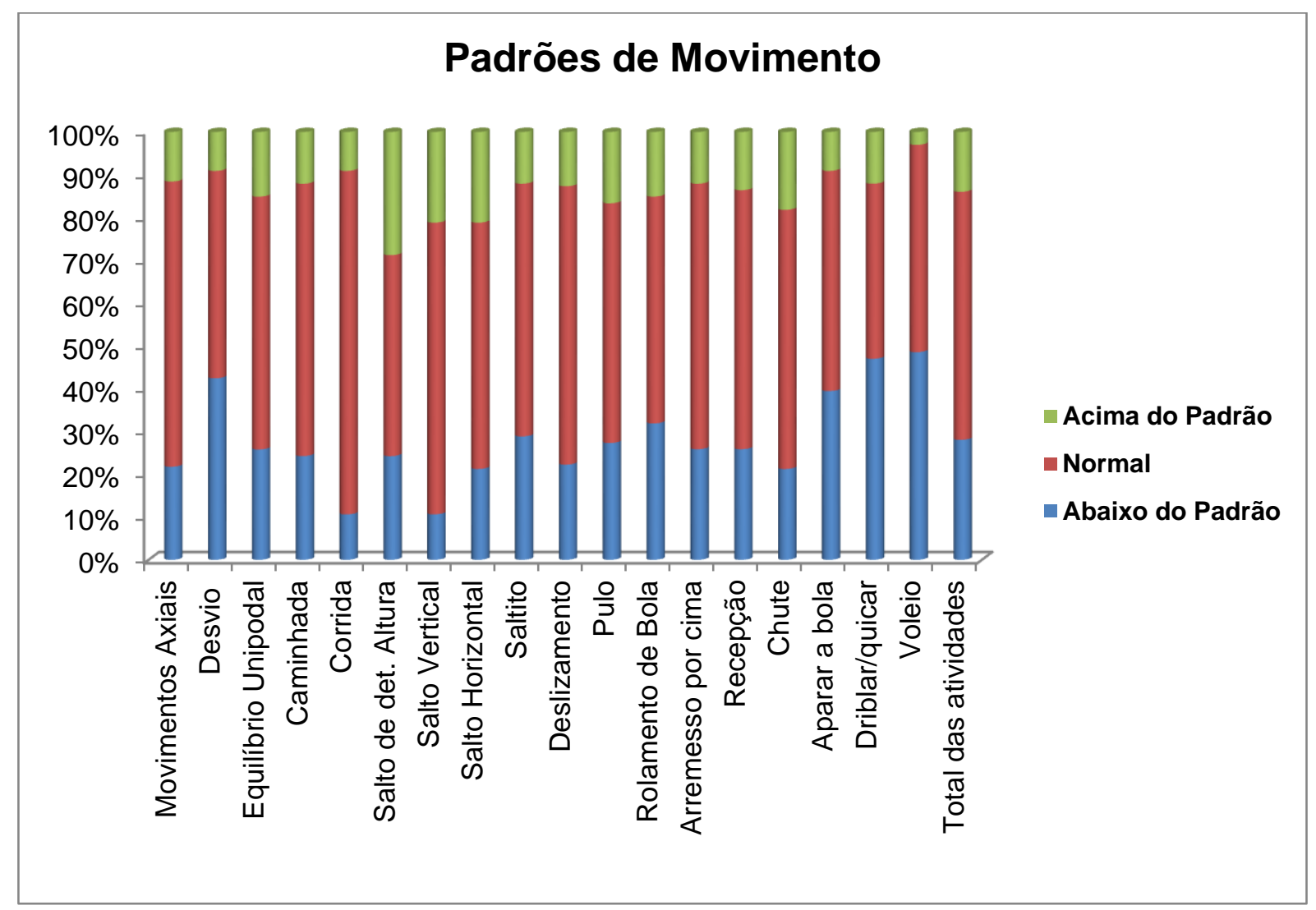

Figura 2 - Percentual da avaliação final do total de alunos avaliados.

$\mathrm{Na}$ avaliação inicial a maioria das atividades realizadas por crianças com 03 e 04 anos apresentou-se no estágio inicial, entre as com 05 e 06 anos prevaleceu o estágio elementar, demonstrando que essas estão abaixo do esperado para cada faixa etária. $\mathrm{Na}$ avaliação final as crianças com 03 e 04 anos alcançaram na maioria das atividades os estágio adequado para a faixa etária, ou seja, o elementar, e as crianças com 05 e 06 anos apresentaram-se na maioria das atividades no estágio maduro, sendo adequado para a faixa etária.

Quanto às visitas para estimulação através do plano de atividades recreativas elaborado pelos participantes da ação de extensão, obteve-se um total de aproximadamente 580 horas 
durante os três anos em que a ação de extensão estava sendo realizadas, divididas igualmente entre as cinco turmas, em torno de 50 minutos semanais para cada uma. O plano de atividades recreativas abordava onze atividades, entre elas: queimada/caçador, pega-pega, boliche, amarelinha, pular corda, basquete com obstáculos, voleibol, chutar pênalti e dois circuitos de tarefas.

\section{CONSIDERAÇÕES FINAIS}

A avaliação inicial do perfil motor demonstrou que havia atrasos no desenvolvimento motor das crianças, visto que grande parte delas encontrava-se abaixo do padrão esperado, independente da faixa etária avaliada. A avaliação final evidenciou que apesar de algumas crianças permanecerem com dificuldades de realizar determinadas atividades a maioria delas alcançou o esperado para a idade em todas as atividades analisadas. Dessa forma podemos afirmar que a estimulação motora foi efetiva. Vale ressaltar, que quanto mais precocemente os atrasos ou alterações motoras forem detectados e estimulados, melhor será a resposta da criança no desenvolvimento motor posterior e maiores serão as chances de revertê-los. Contudo a proposta de estimulação irá sendo alterada conforme a necessidade dos alunos novos. Os objetivos propostos pela ação de extensão foram alcançados, pois, mudanças na proposta curricular político pedagógica da escola foram realizadas visando incluir atividades motoras que estimulassem o desenvolvimento das habilidades motoras.

Assim as ações de extensão devem ser estimuladas e valorizadas, pois estabelecem parcerias e intercâmbios de profissionais das universidades e dos serviços em ambiente propício para que os problemas da prática cotidiana possam ser incorporados às atividades de pesquisa. Da mesma forma possibilitam que os resultados de pesquisas e investigações acadêmicas sejam utilizados para aprimoramento das políticas e práticas dos serviços de saúde e educação.

\section{REFERÊNCIAS}

CAETANO, M. J. D.; SILVEIRA, C. R. A. GOBBI, L. T. B. Desenvolvimento motor de préescolares no intervalo de 13 meses. Revista Brasileira de Cineantropometria e Desempenho Humano, Florianópolis,v. 7, n. 2, p. 05-13, 2005. 
Promoção do desenvolvimento infantil na escola através de atividades motoras recreativas

CASATE, J. C.; CORRÊA, A. K. Humanização do atendimento em saúde: conhecimento veiculado na literatura brasileira de enfermagem. Revista Latinoamericana de Enfermagem, Ribeirão Preto, v. 13, n. 1, p. 105-11, 2005.

FERREIRA, M. C. R.; RAMON, F.; SILVA, A. P. S. Políticas de atendimento à criança pequena nos países em desenvolvimento. Cadernos de Pesquisa, São Paulo, v. 115, p. 65-100, 2002.

GALLAHUE, D. L.; OZMUN, J. C. Compreendendo o desenvolvimento motor: bebês, crianças, adolescentes e adultos. 3. ed. São Paulo: Phorte, 2005.

GODALL, T. 150 propostas de atividades motoras para a educação infantil: (de 3 a 6 anos). Porto Alegre: Artmed; 2004.

KISHIMOTO, T. M. et al. Jogo, brinquedo, brincadeira e a educação. 14 ed. São Paulo: Cortez, 2011.

LOPES, L. O. et al. Associações entre atividade física, habilidades e coordenação motora em crianças portuguesas. Revista Brasileira de Cineantropometria e Desempenho Humano, Florianópolis, v.13, n.1, p. 15-20,2011.

MAFORTE, J. P. G. et al. Análise dos padrões fundamentais de movimento em escolares de sete a nove anos de idade. Revista Brasileira de Educação Física e Esporte, São Paulo,v. 21, n. 3, p. 195-204, 2007.

NOGUEIRA, M. das D. P (org.). Extensão universitária: diretrizes conceituais e políticas. Documentos básicos do Fórum Nacional de Pró-Reitores de Extensão das Universidades Públicas Brasileiras (1987-2000). Belo Horizonte: PROEX-UFMG, 2000. 196 p.

PONTE, C. I. R. V. et al. A extensão universitária na Famed/UFRGS: cenário de formação profissional. Revista Brasileira de Educação Médica, Rio de Janeira, v. 33, n. 4, p. 527 - 534, 2009.

RIZZETTI, D. A.; FABBRIN, A. P. A.; TREVISAN, C. M. Políticas Públicas de Saúde para a criança em Santa Maria-RS. Revista Brasileira em Promoção da Saúde, Fortaleza, v. 22, n. 4, p. 225-232, 2009 .

SANTOS, S.; DANTAS, L.; DE OLIVEIRA J. A. Desenvolvimento motor de crianças, de idosos e de pessoas com transtornos da coordenação. Revista Paulista de Educação Física, São Paulo, v.18, p. 33-44, 2004.

TORQUATO, J. A. et al. Prevalência de atraso do desenvolvimento neuropsicomotor. Revista Brasileira de Crescimento e Desenvolvimento Humano,São Paulo, v. 21, n. 2, p. 259-268, 2011.

WILLRICH, A.; DE AZEVEDO, C. C. F.; FERNANDES, J. O. Desenvolvimento motor na infância: influência dos fatores de risco e programas de intervenção. Revista de Neurociências, São Paulo,v. 17, n. 1, p. 51-56, 2009. 
ANEXO I- Matriz de análise de movimentos (Gallahue e Ozmun, 2005)

\begin{tabular}{|l|l|l|l|l|}
\hline Atividades & Inicial & Elementar & Maduro & $\begin{array}{l}\text { Não } \\
\text { Realiza }\end{array}$ \\
\hline Movimentos Estabilizadores - Axiais & & & & \\
\hline Movimentos Estabilizadores - Desvio & & & & \\
\hline $\begin{array}{l}\text { Movimentos Estabilizadores - Equilíbrio } \\
\text { unipodal }\end{array}$ & & & & \\
\hline Movimentos Locomotores - Caminhada & & & \\
\hline Movimentos Locomotores - Corrida & & & & \\
\hline $\begin{array}{l}\text { Movimentos Locomotores - Salto de } \\
\text { determinada altura }\end{array}$ & & & & \\
\hline Movimentos Locomotores - Salto Vertical & & & & \\
\hline Movimentos Locomotores - Salto Horizontal & & & & \\
\hline Movimentos Locomotores - Saltito & & & & \\
\hline Movimentos Locomotores - Deslizamento & & & & \\
\hline Movimentos Locomotores - Pulo & & & & \\
\hline $\begin{array}{l}\text { Movimentos Manipulativos - Rolamento de } \\
\text { bola }\end{array}$ & & & & \\
\hline Movimentos Manipulativos - Arremesso por & & & & \\
\hline Movimentos Manipulativos - Recepção & & & & \\
\hline Movimentos Manipulativos - Aparar a bola & & & & \\
\hline
\end{tabular}

